\title{
Ventajas epistémicas de la cognición socialmente distribuida*
}

\author{
Anna Estany \\ Universidad Autónoma de Barcelona
}

El objetivo de este artículo es analizar hasta qué punto la cognición (o conocimiento) socialmente distribuida/o incide en la problemática de los valores epistémicos o cognoscitivos 1 . Para ello vamos a proceder del modo siguiente: en primer lugar, plantearemos el problema de los valores epistémicos y cómo lo han abordado algunos de los filósofos de la ciencia, en segundo lugar, analizaremos el enfoque en ciencia cognitiva sobre la Actividad Situada y la Cognición Socialmente Distribuida (CSD), y finalmente, veremos la incidencia de la CSD en la problemática aquí planteada.

Nos encontramos frente a un problema epistemológico que es central en la filosofía de la ciencia, a unas soluciones dadas por los filósofos y a unos problemas pendientes. Dichos problemas pendientes son los que hacen que los filósofos sigan buscando soluciones. La tesis de este artículo es que el modelo cognitivo de la CSD hace aportaciones muy interesantes y ofrece una forma adecuada de abordar esta problemática.

* Una primera versión de este artículo se presentó en el I Congreso Iberoamericano de Filosolía de la Ciencia y de la Tecnología, en Morelia (México), Septiembre del 2000.

Este trabajo ha sido realizado en el marco del Proyecto de Investigación «Anরilisis interdisciplinar de la ciencia», coordinado al Proyecto «Ciencia y valores» (PB980495-C08-06), financiado por el Ministerio de Educación y Ciencia

1 Voy a utilizar "cognoscitivo" en lugar de "cognitivo" en el sentido de valor interno o directamente ligado al conocimiento. La elección de cognoscitivo en lugar de cognitivo es para que no se confunda con el enfoque cognitivo. 


\section{Planteamiento del problema epistémico}

El problema que vamos a analizar se produce por la tensión entre diferentes valores epistémicos a la hora de elegir entre hipótesis y entre fines u objetivos de la ciencia. La filosofía de la ciencia, a lo largo de su historia, ha ido consolidando una serie de indicadores epistémicos que conforman los valores y los fines de la ciencia. Uno de los problemas es que estos valores no pueden llevarse a cabo en la misma medida, lo cual significa que tenemos que priorizar unos frente a otros. Sin embargo. por un lado, no hay un algoritmo para saber en cada momento qué valores hay que poner en primer lugar cuando hay que elegir entre dos teorías en competencia. Por otro lado, la investigación científica requiere la objetividad para que la ciencia tenga fundamento racional. El problema es, pues, cómo aunar la falta de un algoritmo para la elección de teorías y la objetividad de la ciencia. En resumen, cómo puede resolverse el pulso epistémico.

\section{VALORES EPISTÉMICOS}

La filosofía de la ciencia tiene en su haber el análisis de lo que constituyen valores epistémicos. No pretendo dar una enumeración exhaustiva de valores epistémicos pero sí un panorama de cómo filósofos de la ciencia con teorías de la ciencia distintas abordan el tema de los valores, señalando lo que podríamos denominar «indicadores epistémicos».

C. Hempel: valoración de la objetividad de la ciencia

Hempel en «Valuation and objectivity in science» plantea el problema del estatus cognoscitivo de la metodología de la ciencia y de la epistemología. Según Hempel, si no podemos postular la independencia de los valores, la objetividad de la ciencia está en peligro. Hempel no duda de que los factores sociales y políticos puedan influir en los científicos a la hora de investigar en un campo o en otro, en escoger una hipótesis u otra, pero piensa que hay valores independientes y que, en todo caso, si un científico sostiene una creencia para la que carece de evidencia obra irracionalmente.

Según Hempel, hay dos posturas extremas en la cuestión de los criterios objetivos de la investigación científica: el «racionalismo metodológico», según el cual hay unas normas generales fundamentadas a priort por medio del análisis lógico y de la reconstrucción de la racionalidad del conocimiento científico que todo investigador científico tiene que 
cumplir; y el «pragmatismo o naturalismo metodológico», según el cuil los procedimientos científicos deben ser formulados apelando a la actual práctica científica y no a preconcepciones apriorísticas. Estas dos posturas deben tomarse como prototipos extremos entre los que se sitúan los diferentes autores. Resumiendo, dice Hempel:

Entonces, los fundamentos justificatorios para las teorías metodológicas. tanto la variedad naturalista como la analítica-explicativa tienen una faceta descriptiva y una faceta que refleja la valoración epistemológica. Ninguno de los dos enfoques de la metodología de la ciencia es puramente a priori, ni puramente descriptivo. (Hempel, 1983: 87)

Para la valoración epistemológica Hempel introduce los «desiderata», como constreñimientos para una teoría de la decisión entre teorías. Los desiderata tienen el carácter de normas o valores epistemológicos pero sólo pueden ser formulados vagamente, por lo que no determinan unívocamente lá elección entre teorías. En particular, no proporcionan un algoritmo que, análogamente a los algoritmos matemáticos. determinan una única solución para cada problema dentro de su dominio. Así, la elección de teorías hecha por científicos individuales comprometidos con los desideratas están también influenciados por factores que pueden diferir de persona a persona. Estas diferencias pueden referirse tanto a las prioridades de los desiderata como a factores idiosincráticos y subjetivos. La cuestión es si y hasta qué punto esto afecta a la objetividad de la ciencia. A lo cual contesta Hempel:

Pienso que pueden ofrecerse razones plausibles que apoyan una reconstrucción racional objetiva pero «relajada» de acuerdo a la cual los procedimientos científicos están gobernados por normas metodológicas algunas de las cuales son explícitas y precisas, mientras que otras son vagas. Los requisitos de clausura deductiva y de consistencia lógica para teorías aceptables serían del primer tipo, mientras que otros desiderata que gobiernan la elección de teorías serían del segundo (Hempel, 1983: 93).

Así pues, Hempel concibe la ciencia como una empresa objetiva, con una serie de requisitos impuestos por ciertas normas generalmente admitidas. Sin embargo, reconoce que la ciencia empírica, a veces, emplea conceptos que están caracterizados sólo vagamente, aunque no tienen por qué ser arbitrarios y subjetivos. De esta forma excluye así una visión de la ciencia como una empresa en que «todo vale». 


\section{B. Van Fraassen: Virtudes de una teoría}

Van Fraassen señala que hay características genuinas de las teorías, como la coherencia y la adecuación empírica y otras virtudes como la elegancia matemática y la simplicidad cuya observancia tiene que ver con factores contextuales. Esta parte de intereses sería la dimensión pragmática que interviene en la valoración y aceptación de teorías, serían «virtudes pragmáticas». (Van Fraassen, 1980:87-88) Tanto las genuinas como las pragmáticas constituyen valores epistémicos pero Van Fraassen no da criterios para saber qué virtudes hay que priorizar.

\section{A. Goldman: Criterios epistemológicos evaluadores}

Goldman en Epistemology and Cognition propone unos valores epistémicos que pueden actuar como criterios a la hora de sopesar hastit qué punto tenemos justificación para nuestras creencias. Estos valores son fiabilidad, poder y velocidad. La fiabilidad tiende a producir el mayor número posible de creencias verdaderas, el poder consiste en la capacidad de producir creencias verdaderas y la velocidad es la rapidez con la que pueden producirse creencias verdaderas. En este caso se proponen tres valores como fundamentales pero no se resuelve el problema aquí planteado ya que aún nos queda determinar cuál de estos valores priorizamos sobre los demás. Es fácil imaginar situaciones en la investigación científica en que haya que sacrificar algo de fiabilidad en aras de una mayor rapidez, o viceversa.

\section{Criterios racionales para la elección de teorías}

Los criterios de elección racional constituyen uno de los elementos de los modelos de cambio científico. El establecimiento de estos criterios requiere el contexto de la justificación y aquí es donde entran los valores epistémicos. Veamos algunos de los valores epistémicos propuestos por autores de modelos de cambio científico.

T.Kuhn: Un difícil equilibrio entre valores objetivos y subjelivos

Kuhn en La estructura de las revoluciones cientificas, 1962, habla poco de valores epistémicos, aunque sin negarlos porque, como dirá más tarde, había que poner el énfasis en los factores subjetivos y externos, ya que los objetivos e internos habían sido ya suficientemente tratados. Pero en 1973, Kuhn publica el artículo «Objectivity, value judgement 
and theory choice», en que sí habla directamente de valores epistémicos y del papel que juegan en la elección de teorías en competencia. Kuhn parte del supuesto de que no es posible producir un algoritmo capaz de determinar la elección correcta. Esto hace que los científicos no puedan evitar la intervención de elementos subjetivos para determinar qué criterios elegir. Y, aún suponiendo que existiera un algoritmo para la elección de teorías, cada individuo tendría el suyo. Kuhn expresa su propuesta en los términos siguientes:

Mi argumento ha sido dirigido, hasta ahora, hacia dos puntos. En primer lugar, proporciona la evidencia de que las elecciones que los científicos realizan entre teorías en competencia dependen, no sólo de los criterios compartidos, aquellos que mis críticos llaman objetivos, sino también de factores idiosincráticos dependientes de la biografía y personalidad individual (Kuhn, 1977:329)

Kuhn no duda en reconocer la existencia de unos valores cognoscitivos compartidos para la elección de teorías, tales como el rigor, la consistencia, el campo de aplicación, la simplicidad y la fecundidad; y que una teoría que posea estas características será preferida a la que no las tenga o a la que las tenga en menor grado. Sin embargo, para Kuhn estos criterios de elección racional no funcionan como reglas que determinan unívocamente la elección sino como valores que influyen en dicha elección. Así, dice Kuhn, dos científicos pueden compartir los mismos valores pero al mismo tiempo pueden elegir teorías distintas en un momento determinado. Por lo tanto, los criterios o valores pueden fijarse pero varían a través del tiempo y de un individuo a otro en función de cómo se pongan en práctica y de qué peso se les asigne, y esto depende de la idiosincrasia de cada científico y de las condiciones externas (sociales, políticas, culturales, etc.). En este sentido, el epistemólogo debería interesarse por los rasgos de personalidad y los contextos sociales que más favorecen la empresa científica, es decir, cuáles son las circunstancias personales y sociales que en mayor medida permiten preservar los valores cognoscitivos.

W.H.Newton-Smith: la explicación de los fenómenos como objetivo prioritario

Newton-Smith (1987) señala como objetivos de la ciencia buscar las verdades, aunque no cualquier verdad, y explicar los fenómenos. A su vez, señala Ios criterios que hay que tener en cuenta al elegir la teoría 
que mejor explicará los procesos del mundo: el éxito observacional, que será nuestro indicador primario de incremento de verosimilitud; la fertilidad, que implica tener posibilidades de desarrollo futuro; el historial, es decir; cuánto tiempo ha mantenido el éxito observacional; el apoyo interteórico, esto es, poder contar con el apoyo de otras teorías que han probado que son exitosas; la adaptabilidad, o lo que es lo mismo, poder ajustar los problemas o fracasos puntuales; la consistencia interna, que implica que no haya afirmaciones que se contradigan mutuamente; la compatibilidad con creencias metafísicas bien fundadas; y por último. la simplicidad, de la que habría que especificar sus características (Newton-Smith, 1987:245-249)

De alguna forma, todos los autores que han abordado la dinámica científica se han referido a los criterios para la elección de teorías. Cuando Lakatos (1983) señala las características para que un programa de investigación sea progresivo y no regresivo está dando criterios para elegir entre teorías en competencia. Cuando Popper (1962) dice que uni buena teoría tiene que ser falsable, también establece un criterio. Idem cuando Toulmin (Toulmin 1977: 215) señala los modos de resolver problemas: refinando la terminología, introduciendo nuevas técnicas de representación o modificando los criterios para identificar casos a los que sean aplicables las técnicas corrientes. Y cuando Laudan dice que hay que tomar las teorías que resuelvan el mayor número de problemas, también nos da un criterio. Por su parte, P.Kitcher establece una serie de indicadores, que hay que tener en cuenta al evaluar la producción teórica de una disciplina, tales como el lenguaje utilizado por el científico, las cuestiones significativas, los enunciados sobre el objeto de estudio, los patrones o esquemas explicativos, los criterios de credibilidad, los paradigmas de experimentación y observación y los ejemplares, correctos o incorrectos, de razonamiento. Pues bien, todos estos indicadores son criterios o valores epistémicos que actúan como normas a tener en cuenta en la práctica científica (Kitcher, 1993: 74).

\section{LA CUESTIÓN DE LA PRIORIDAD DE VALORES Y FINES}

A pesar de todas las aportaciones de los filósofos a los valores epistémicos, el problema planteado al principio de este artículo sigue en pie, yá que existe la cuestión de cómo establecemos prioridades. ¿Poseemos algún algoritmo por el que podamos asignar pesos a los distintos indicadores y así determinar unívocamente qué criterio es el prioritario en cada momento de decisión entre teorías? Ninguno de los fílósofos aquí presentados apuesta por la existencia de un algoritmo. Tampoco 
tenemos jerarquías fijadas de antemano, aunque en determinados momentos históricos haya habido modelos metodológicos que han primado uno u otro valor epistémico.

Tomando en consideración lo expuesto anteriormente podemos satcar las consecuencias siguientes:

1) No hay un algoritmo para la elección de teorías porque no hay un algoritmo para la elección de criterios para elegir teorías.

2) La investigación científica es una práctica científica realizada por individuos con intereses y fines idiosincráticos inmersos en contextos socioculturales, y el resultado es que estas diferencias se plasman en la actividad cognitiva y rezuman en el quehacer científico.

3) La ciencia no puede desprenderse de la objetividad, de la generalidad, de la sistematicidad, si queremos que nuestro conocimiento tenga fundamento racional.

4) La cuestión es cómo aunar, por un lado, la constatación de 1) y 2) y, por otro, el imperativo de 3).

Desde la filosofía de la ciencia se han dado algunas respuestas a esta problemática. Veamos algunas de las más importantes.

Larry Laudan: del modelo jerárquico al modelo reticular

L. Laudan en Science and Values, 1984, analiza el papel de los valores cognoscitivos en la racionalidad científica. Rechaza lo que él denomina «el modelo de estructura jerárquica», según el cual cuando hay desacuerdo en el terreno de los hechos se recurre a las reglas metodológicas compartidas y, cuando hay desacuerdo respecto a las reglas metodológicas se recurre al nivel axiológico, en el que se encuentran los fines o principios compartidos por la ciencia. Laudan cree que este modelo no soluciona todos los casos de desacuerdo, aunque no descarta que pueda ser útil en algunos casos. La alternativa que Laudan ofrece al modelo jerárquico es lo que él llama «el modelo reticular de la racionalidad científica» en el que el sistema axiológico, la metodología y los enunciados factuales están conectados por relaciones de mutua dependencia.

Newton-Smith: la metáfora del enólogo

Otro de los filósofos que aborda la relación entre valores epistémicos y entre éstos y los fines de la ciencia es Newton-Smith, quien compara 
al científico con el mezclador de vinos (Newton-Smith; 1987). El mez-clador de vinos tiene unos conocimientos sobre vinos, unos criterios sobre lo que es y no es un buen vino, pero no tiene un catálogo de cómo y qué cantidad debe mezclar de cada uno. Al científico le ocurre algo parecido. Todos los científicos comparten los valores antes enunciados sobre una buena teoría, pero a la hora de combinar la simplicidad con el éxito observacional, con el apoyo teórico, etc. entra en juego la intuición, algo que Newton-Smith considera que juega un papel importante en la actividad científica.

\section{P.Kitcher: hacer de la necesidad virtud}

Kitcher critica una concepción del método científico defendida por «La leyenda» (refiriéndose al empirismo lógico) y caracterizada por los supuestos siguientes: i) es imposible que dos científicos sostengan dos proposiciones incompatibles frente a una evidencia total sobre un fenómeno; ii) frente a los mismos estímulos los científicos reaccionan de la misma forma: el sistema cognitivo de un científico — cualquier científico-_ contiene el mismo conjunto de fines y el mismo conjunto de propensiones inferenciales para trabajar sobre inputs de la experiencia; iii) los científicos trabajan bien y sus fracasos son debidos a ignorancia o información errónea.

Frente a esta concepción, Kitcher propone un enfoque alternativo basado en cuatro tipos de variación cognitiva:

1) Si los científicos tienen acceso a diferentes cuerpos de información, entonces pueden tener diferentes creencias con lo cual los contenidos de la memoria declarativa (y posiblemente del conjunto de los fines a largo término y de la memoria procesual) diferirán de un científico a otro.

2) Posiblemente las proposiciones se almacenarán de formas distintas en la memoria declarativa de los científicos, por tanto, lo que es fácilmente accesible para un científico no lo es para otro.

3) Dependiendo del orden y la frecuencia de la exposición a diferentes tipos de información, algunas de las propensiones pueden ser más probablemente activadas que otras. Dos científicos pueden compartir las mismas propensiones, en el sentido de que en ambos está presente el mismo repertorio, pero optar por una u otra en función de diferentes desarrollos intelectuales.

4) Los científicos pueden diferenciarse en los contenidos de su memoria procesual, dándose propensiones inferenciales en uno que no se encuentran en otro (Kitcher, 1993: 61-65) 
La conclusión que saca Kitcher de esta variación y del hecho de que ningún científico individual puede almacenar todas tas proposiciones que son relevantes para el avance de un campo de investigación, es que las diferencias entre los científicos no son accidentales sino esenciales para el progreso científico: el desarrollo del campo se atrofiaría si se impusiera la uniformidad. O sea que la variación es beneficiosa para el progreso científico.

Kitcher hace una comparación con la variación biológica en el sentido de que también es beneficiosa, ya que la variación en poblaciones naturales puede hacer que un linaje sobreviva a acontecimientos que en una población monomórfica se hubiera extinguido. Sin embargo, de la misma forma que el reconocimiento de la variación biológica no nos impide reconocer que ciertos alelos (y sus portadores) son mayormentc no funcionales, tampoco nuestra apreciación de la variación cognitiva nos priva de reconocer que ciertos tipos de sistemas cognitivos son (o serían) incompetentes.

\section{LA ACTIVIDAD SITUADA Y LA COGNICIÓN SOCIALMENTE DISTRIBUIDA}

Si aceptamos la propuesta de Kitcher de que la variación es beneficiosa para el progreso científico y que una de las razones es que ningún científico individual puede almacenar toda la información relevante para la investigación científica de un fenómeno, es de suma importancia estudiar las posibilidades reales, las cuales dependen de las capacidades cognitivas de los humanos, es decir, de que nuestro cerebro esté diseñado de tal forma que pueda haber transferencia de información entre los científicos, lo cual hará posible que los científicos puedan aunar esfuerzos que revertirán en el progreso científico.

Uno podría decir que para esto están los congresos, las revistas científicas y el internet, por poner sólo algunos ejemplos de medios de comunicación entre los científicos. Pero los beneficios epistémicos de la variabilidad cognitiva son previos a la publicación de los resultados de la investigación científica, es decir, están en el momento mismo de la investigación, en los equipos de científicos que están trabajando codo con codo en los laboratorios. Los beneficios de la variabilidad cognitiva (si los hay) empiezan antes de que la investigación científica tome cuerpo y se haga pública. Y es en este punto donde la idea de la cognición socialmente distribuida es absolutamente pertinente para que la variabilidad cognitiva dé sus frutos.

La idea de la cognición socialmente distribuida (o conocimiento socialmente distribuido) (CSD) ha surgido ligada a la idea de la activi- 
dad situada (AS) (Suchman, 1987), la cual se entiende como la interacción de los sujetos con los artefactos e instrumentos en determinadas circunstancias sociales. La AS consiste en integrar todos los factores que intervienen en una situación determinada. Esta idea puede encontrarse en autores procedentes de campos disciplinares distintos, por ejemplo. en.W. J. Clancey (1993) procedente de la Inteligencia Artificial, en L. Suchman (1993) de la Etnometodología, en J. Lave (1988)de la Etnografía cognitiva, etc. Tal como apunta C. Lozares (en prensa, Papers) la AS constituye más un vivero de preocupaciones e ideas que un cuerpo doctrinal establecido. Lo cual no es óbice para que sea un enfoque importante y muy prometedor, tanto para la ciencia cognitiva como para campos relacionados con procesos cognitivos.

Por su parte, la CSD consiste en que las percepciones, representaciones, memoria y, en general, las proyecciones y procesos cognitivos están generados, extendidos y distribuidos diferencialmente entre los artefactos y entre los agentes debido a que en el proceso de interacción se entabla un necesario reconocimiento y representación mutua, una interlocución, intercambio, negociación y apropiación de los procesos cognitivos.

Tanto la AS como el CSD constituyen una alternativa al pensamiento simbólico propio de la IA clásica. En una perspectiva clásica las diferencias de los contenidos cognitivos y del poder inferencial entre los individuos y grupos se atribuye a diferencias en la capacidad de razonar. Pero si se vincula el conocimiento a situaciones y contextos que influyen en su producción, tales como el conocimiento de otros sujetos, los instrumentos, la interacción entre individuos, etc. se comprende que los resultados cognitivos estén representados, memorizados y procesados de manera distribuida entre los sujetos que intervienen. Este nuevo enfoque (AS Y CSD) considera que cuando los humanos razonamos lo hacemos a partir de esquemas culturales aprendidos socialmente, de la pertenencia a un grupo, de la interacción con otros individuos a través del lenguaje, es decir, a partir de referencias culturales comunes que actúan como premisas del razonamiento e interfieren en el desenlace de la inferencia (D'Andrade, R. 1989).

Este enfoque se ha tomado para analizar diferentes actividades. Agree y Shrager (1991) analizan la actividad de fotocopiar en la que interviene un sujeto y un artefacto. Aquí la interacción principal no es la social con otros actores sino entre un sujeto y la fotocopiadora. Para Agree y Shrager el contexto consiste en la construcción cultural de la reificación de la velocidad productiva. Y consideran que este modelo de interacción puede aplicarse también a otros contextos industriales. A. Cicourel (1994) 
toma este enfoque para analizar diferentes marcos institucionales. El marco institucional incluye la experiencia de los participantes que trabajan juntos, las representaciones mutuas de los agentes, las responsabilidades recíprocas, etc. La posición del individuo en la estructura institucional (sea en las divisiones funcionales o sociales, sea en las verticales u horizontales) influye en el acceso a la información, en el apoyo y consenso del personal y en la comunicación interna y externa.

\section{Edwin Hutchins: de LA COGNICIÓN INDIVIDUAL a LA COGNICIÓN SOCIAL}

Vamos a analizar el enfoque de la CSD a través de la obra de E. Hutchins, uno de los principales promotores del conocimiento socialmente distribuido en el campo de las ciencias cognitivas.

La tesis de Hutchins está desarrollada, principalmente, en su obra Cognition in the Wild (1995). Hutchins procede de la antropología cognitiva. Sus campos de estudio han sido la navegación y la aviación, actividades a las que personalmente es muy aficionado.El libro del 95 tiene su origen en su estancia en 1980 en el «U.S.Navy ship» pero el estudio empezó más tarde, en 1984 cuando le ofrecieron trabajar para la «Navy Personnel Research and Development Center in San Diego» con el título de «Personnel Research Psychologist». Se le dio carta blanca para estudiar lo que él considerara interesante. En aquel momento estudió lo que él mismo llamaba «cognición situada en su medio natural» (naturally situated cognition) pero más adelante, una vez completado su primer período de estudio, pasó al estudio de la «cognición socialmente distribuida».

Hutchins señala que con la revolución cognitiva la antropología llevó a cabo ciertas innovaciones: en un primer paso, se apartó de la sociedad para investigar el conocimiento que un individuo tiene que tener para funcionar como miembro de una cultura; en un segundo paso, se apartó de la práctica en un intento de aprender lo que la gente conoce. Sin embargo, los antropólogos perdieron el hilo tanto de cómo la gente conoce lo que conoce como de la contribución de los entornos en los que el conocimiento se lleva a cabo. Hutchins considera que ahora que la disciplina está ya asentada hay que abandonar estos supuestos ya que son una carga para entender la naturaleza de la cognición humana. Dice Hutchins:

La definición ideacional de la cultura nos impide ver que los sistemas de cognición socialmente distribuida pueden tener, en sí mismos, propiediades cognitivas interesantes. En la historia de la antropología, no hay ape- 
nas un concepto más interesante que la división del trabajo. En términos del presupuesto de energía de un grupo humano y de eficiencia con la que un grupo explota su entorno físico, los factores sociales de organización a menudo producen propiedades de grupo que difieren considerablemente de las propiedades individuales. Claramente, el mismo tipo de fenómenos ocurren en el dominio cognitivo. Dependiendo de su organización, los grupos deben tener propiedades cognitivas que no son previsibles desde un conocimiento de las propiedades de los individuos en el grupo. El énfasis por encontrar y describir «estructuras de conocimiento» que están en algún lugar «dentro» del individuo fomenta que pasemos por alto el hecho de que la cognición humana está siempre situada en un complejo mundo sociocultural y no puede no ser afectada por él. (Hutchins, 1995: xiii)

Lo que plantea Hutchins es que hay que reconsiderar la relación entre la cognición vista como una actividad mental solitaria y la cognición vista como una actividad socialmente situada. Su obra seminal Cognition in the Wild es un intento de poner la cognición en el mundo social y cultural. Lia cognición «en su medio natural» (in the wild) se entiende como cognición fuera del laboratorio. Lo que quiere demostrar es que la cognición humana no es solamente que esté influenciada por la cultura y la sociedad sino que es un proceso cultural y social. Para ello quiere sacar las unidades cognitivas de análisis fuera de la piel de la persona individual y tratar el grupo como un sistema computacional y cognitivo.

Dos de los estudios de caso a través de los cuales Hutchins argumenta a favor de la CSD son el equipo de un buque de la marina naval de los Estados Unidos y el de la cabina de un avión. Como señala $M$. Salomon en una reseña del libro de Hutchins (Philosophy of Science, v.64, nº 1 , Marzo, 1997) el estudio etnográfico de este equipo de navegación le sirve como argumento a favor de las siguientes conclusiones teóricas: el cerebro no es, en general, la unidad de cognición, por tanto, las tareas cognitivas se llevan a cabo por entidades más amplias tales como el cuerpo entero o el cuerpo entero más herramientas o una comunidad de personas integradas en esta actividad; la cognición no es, en general, manipulación de símbolos gobernados por reglas formales explícitas sino propagación gradual de propiedades funcionales organizadas a través de un conjunto de medios maleables, siendo este proceso constreñido por la conducta de otros (que corrigen al novicio) o por el propio monitor del que realiza la tarea; y cultura y cognición no son separables ya que la cultura determina los detalles de cómo una tarea cognitiva es representada e implementada. 
El caso de la cabina de avión lo expone Hutchins en un artículo con T. Klausen «Distributed cognition in an airplane cockpit» (1996) donde estudia los procesos cognitivos que tienen lugar entre los miembros del equipo que dirige un vuelo. Dice que como pasajeros lo que nos debe preocupar no es tanto si el piloto va a hacerlo bien sino si el sistema formado por los pilotos y la tecnología del entorno de la cabina lo están haciendo bien. Es la actuación del sistema y no las habilidades de un piloto individual lo que determina si un pasajero vivirá o morirá. La unidad de análisis debe permitirnos describir y explicar las propiedades cognitivas del sistema de cabina que está compuesta por los pilotos y su entorno informacional. A esta unidad de análisis le llama «un sistema de cognición distribuida».

Tomando una cabina de piloto como un sistema cognitivo se puede decir que el procesamiento de información en un sistema distribuido puede ser caracterizado como una propagación de un estado representacional a través de medios representacionales. En la cabina, algunos de los medios representacionales están situados dentro de las tramas individuales. Otros, tales como el habla están localizados entre los pilotos, y aún hay otros que están en la estructura física de la cabina. Cada medio representacional tiene propiedades físicas que determinan la disponibilidad de representaciones a través del espacio y del tiempo y el tipo de procesos cognitivos requerido para propagar el estado representacional dentro o fuera de este medio. Cambios en el medio de representación de la información de la tarea relevante o en la estructura de representaciones en un medio particular pueden,por tanto, tener importantes consecuencias para el comportamiento cognitivo del sistema de cabina.

La circulación de información a través del sistema tiene consecuencias para la formación de expectativas y modelos de la situación del avión. Estas expectativas y modelos organizan la conducta de la tripulación y, cuando son compartidas, permiten a los miembros de la tripulación coordinar sus acciones con los demás. Además, la circulación de información entre los miembros de la tripulación depende, a veces, de las valoraciones que los miembros de la tripulación hagan de sus propios estados de conocimiento y de la de los otros. La relación entre las propiedades cognitivas del sistema de cabina de un piloto, en tanto en cuanto está determinada por la circulación de representaciones y las propiedades cognitivas de los pilotos individuales es, por tanto, muy compleja.

A lo largo del análisis de este caso, Hutchins identifica un conjunto de posibles rutas para la información del sistema de cabina del piloto durante los acontecimientos de manejo a través del espacio por el Control de Tráfico Aéreo (ATC). Algunas de estas rutas observadas son aque- 
llas que son anticipadas por el diseño del sistema. Otras, que no estaban previstas en el diseño del sistema, contribuyen, sin embargo, a su actuación. Aunque nunca podemos saber por adelantado qué ruta particular para la información se utilizará, el análisis de este acontecimiento establece un tipo de prueba existente para las rutas observadas. Tal como hemos visto, hay muchas rutas posibles para la información en este sistema. En algunos casos las rutas son redundantes, así si una se bloquea, la información para la tarea relevante puede proceder por otra vía. Esta redundancia parece contribuir a la robustez del sistema en el caso de fracasos locales.

Ciertamente, las propiedades del sistema de cabina del piloto están determinadas en parte por las propiedades cognitivas de los pilotos individuales. Están también determinadas por las propiedades físicas de los medios representacionales a través de los que un estado representacional de una tarea relevante es propagada, por la organización específica de las representaciones asentadas en estos medios, por las interacciones de metarepresentaciones sostenidas por los miembros de la tripulación, y por las características distribucionales del conocimiento y acceso a la información de la tarea relevante a través de los miembros de la tripulación. Entender las propiedades de la cognición individual es, por tanto, sólo un primer paso en un esfuerzo para entender cómo operan estos sistemas cognitivos humanos más complejos.

\subsection{LA ESTRUCTURA COGNITIVA DE LA COMUNICACIÓN}

No cabe duda de que todos los temas tratados por Hutchins son relevantes para la epistemología, sin embargo, para el problema aquí tratado, lo es especialmente el de la comunicación entre los miembros del grupo, relacionada con la organización de dicho grupo. Hutchins argumenta que la amplitud de la comunicación disponible para los miembros de un equipo de navegación afectará a las propiedades computacionales del equipo tomado éste como un sistema cognitivo. Y dado que la mayor parte de la comunicación es verbal, las propiedades del lenguaje serán relevantes para que la computación se lleve a cabo. Sin embargo, hay que tener en cuenta que el lenguaje se ve de otra forma al poner el énfasis en un sistema de cognición socialmente distribuida en lugar de ponerlo en el individuo. Las propiedades del lenguaje interactúan con las propiedades de la tecnología de la comunicación de tal forma que afectan a las propiedades computacionales del sistema cognitivo. 
La idea es que las propiedades cognitivas de los grupos pueden diferir de las de los individuos que constituyen el grupo. Ahora bien, una cosa es afirmar una idea y otra cosa muy distinta es demostrarlo. Hutchins dice que los acontecimientos en el mundo real son siempre complicados de analizar pero tenemos ahora otro tipo de demostración que es la simulación. Hutchins (1995: cap.5) describe una simulación computacional que explora el papel de la comunicación en la producción de las propiedades cognitivas del grupo. Para ello se centra en una propiedad cognitiva particular, sobre la cual todo el mundo está de acuerdo que es una propiedad de la cognición individual, y después desarrolla una forma de mostrar que si esta propiedad se manifiesta en el grupo, la forma en la que se manifiesta depende de la organización social del grupo.

La propiedad analizada se conoce como «sesgo de la confirmación» («confirmation bias»). El sesgo de la confirmación influye en la interpretación de los fenómenos que percibimos. Se define como la propensión a afirmar anteriores interpretaciones y a ignorar o reinterpretar la evidencia que va contra una interpretación ya formada. Es una propensión para confïrmar una hipótesis que uno tiene sobre la naturalezal del mundo. Esta propensión es ampliamente aceptada como una propiedad de la cognición individual. El problema con el sesgo de la confirmación es que impide a un organismo explorar otras interpretaciones posibles. En ausencia de comunicación las interpretaciones formadas por las redes individuales (que cada una exhibe su propio sesgo de confirmación) depende de tres parámetros que caracterizan dichas redes: la estructura del constreñimiento subyacente, el acceso a la evidencia procedente del entorno, y los patrones iniciales de activación. Si una comunidad está compuesta de individuos que difieren en algunos de estos parámetros. entonces es probable que dichos miembros lleguen a diferentes interpretaciones. Por tanto, la diversidad de interpretaciones es bastante fácil que se produzca siempre que la comunicación entre los miembros de la comunidad no sea excesiva.

Lo que plantea Hutchins es qué factores pueden producir esta propiedad en sistemas de cognición distribuida. Espera mostrar que las consecuencias de esta propiedad de la cognición individual para las capacidades cognitivas de grupos de humanos depende casi enteramente de cómo el grupo distribuye las tareas cognitivas entre sus miembros. Es decir, algunas formas de organización de tareas cognitivas llevan a la exacerbación de aspectos maladaptativos de esta propiedad de los sistemas mentales, mientras que otras formas de organización pueden convertir en una virtud adaptativa a nivel de grupo lo que era un vicio a nivel individual. 
Hay muchas actividades humanas regidas por sistemas con múltiples actores que buscan un interpretación coherente de una serie de fenómenos. El equipo de navegación y el de la cabina de un avión son dos ejemplos, pero también cualquier comunidad de científicos es un sistema de este tipo. Dar una interpretación de una serie de fenómenos es una instancia de lo que los científicos computacionales llaman un «problema de satisfacción de constreñimientos» («constraint-satisfaction problem»). Una buena interpretación es aquella que es, a la vez, internamente coherente y de acuerdo con los datos disponibles. Hutchins para simular la formación de interpretaciones utiliza una red conexionista denominada «constraintsatisfaction network», tomada de Rumelhart et al. (1986). Hutchins es consciente de que todas las simulaciones son, por necesidad, una simplificación de los fenómenos que modelan. En el presente caso, muchos de los importantes hechos sobre la comunicación real en sistemas humanos no están representados, sin embargo y a pesar de estas limitaciones, la simulación clarifica una serie de cuestiones que hasta el momento estaban en la oscuridad.

\subsection{Organización SOCIAL Y PROPIEDAdES COGNITIVAS DE LOS GRUPOS}

Tal como se ha indicado, una de las tesis de Hutchins relacionada con la comunicación es que la organización social puede influir en las propiedades cognitivas del grupo y, en consecuencia, sobre el valor epistémico del conocimiento generado por el grupo.

Puede haber ocasiones en que los sesgos se acentúen con el grupo, sobre todo cuando la información es muy parecida y continua entre todos los miembros. Esto indica que no siempre cuanta más información mejor. A veces, incrementar la información puede ocasionar propiedatdes no deseables a nivel de grupo. Esto se ve en algunas simulaciones pero también hay casos reales como el que cuenta Buckhout (1982) que preguntó a un grupo de personas que describieran un crimen del que habían sido testigos y la información creció enormemente pero también la cantidad de errores. Este es un caso en que todos los miembros de un grupo se afianzan en una interpretación más fuertemente de la que se habrían afianzado cada uno por separado. Pero el caso en que todos los miembros del grupo coinciden en la misma interpretación no es muy habitual. Hutchins presenta casos donde hay una distribución interesante de acceso a la evidencia, donde la fuerza de la comunicación es moderada, donde los patrones tienen una interconectividad parcial (esto es, donde los individuos no hablan sobre todo lo que conocen, sólo sobre 
las cosas importantes) y donde la comunicación no es continua. De esta forma se evita el problema de que los sesgos se acentúen en el conjunto del grupo.

Algunas instituciones necesitan garantizar que sus miembros llegarán a una interpretación compartida en un tiempo razonable. Hay varias formas de llegar a esta interpretación compartida y de resolver, así, la diversidad de interpretaciones: jerarquía (con o sin comunicación), consenso (por unanimidad o nada, o por voto).

Muchas instituciones, en la realidad, toman alguno de estos métodos para llegar a una interpretación compartida. La organización social, o más precisamente, la distribución de poder para definir situaciones como reales, determina la localización de un sistema cognitivo en un espacio de intercambio. Cuando el poder está distribuido en una estructura «horizontal» hay más probabilidades de una diversidad de interpretación y más indecisión. Donde el poder tiene una estructura «vertical» es justo lo contrario: menos diversidad de interpretaciones y de indecisión.

Cuando hay necesidad tanto de exploración de un espacio de interpretación como de consensus de interpretación, un sistema tiene dos modos de operar:

1) Renunciar a la habilidad de alcanzar una decisión en favor de la diversidad de interpretación. Los participantes en el sistema proceden en relativo aislamiento y en paralelo. Cada miembro puede estar sujeto al sesgo de la confirmación, pero como proceden de forma independiente, el sistema en su conjunto no exhibe sesgo de confirmación.

2) Romper el aislamiento de los participantes y exponer las interpretaciones a evidencia que las desconfirma a fin de que se evite perseverancia errónea de una interpretación cuando hay disponible una mejor. Este modo de proceder renuncia a la diversidad en favor del compromiso a una única interpretación que significará la nueva realidad de la situación.

Resumiendo, podemos decir que Hutchins propone una hipótesis sobre la relación entre el tipo de organización y las propiedades cognitivas del grupo y recurre a la simulación como recurso computacional para demostrar su hipótesis, aun reconociendo las limitaciones y simplificaciones que supone este método de comprobación de hipótesis. 
7. INCIDENCIA DEL CONOCIMIENTO SOCIALMENTE DISTRIBUIDO EN LA PROBLLEMÁTICA DEL PULSO EPISTÉMICO

Que el enfoque de Hutchins sobre la cognición humana es relevante para la fundamentación del conocimiento, está fuera de duda. Lo que Hutchins dice de la tripulación de un barco o de un avión puede aplicarse a un equipo de científicos trabajando en el laboratorio. En esta misma dirección apunta M.Salomon en una reseña del libro de Hutchins:

Las afirmaciones de Hutchins de que la cognición social puede ser más robusta y maleable (porque la «expertez» está distribuida y solapada) y más exitosa (porque dispone de mejores medios de llegar al consenso) nos puede llevar a nuevas sugerencias normativas para la práctica científica. (Salomon: Philosophy of Science, v.64, nº, Marzo, 1997)

Hacer un estudio de caso como los de Hutchins pero aplicado a tun equipo de investigación va más allá de las pretensiones de este artículo. El objetivo, mucho más modesto, es analizar algunos problemas cognoscitivos provocados por el pulso epistémico en la elección de teorías en competencia y ver cómo pueden clarificarse a la luz de los supuestos teóricos de la CSD.

Base científica de la variabilidad cognitiva

Muchas de las ideas propuestas por los enfoques de la AS y de la CSD pueden parecer triviales, de sentido común y no novedosas. Como ya he indicado, aunque los temas no son nuevos sí lo son muchas de las ideas con las que se abordan estos temas. Sin embargo, lo más interesante del modelo de Hutchins es que proporciona base empírica (neurobiológica, psicológica y computacional) a la idea de que la variación cognitiva tenga efectos benéficos para el conocimiento. Hutchins presenta un modelo cognitivo que hace plausible psicológicamente la colaboración cognitiva. Y esto es fundamental porque, por mucha voluntad de cooperación que tuviéramos, si nuestro sistema neurobiológico no tuviera esta capacidad, de nada serviría el voluntarismo.

Uno de los factores más convincentes de Hutchins procede de asentarse en uno de los enfoques más prometedores de la ciencia cognitiva. el conexionismo. Es posible que el nivel que se ha alcanzado en la simulación del conocimiento socialmente distribuido no sea muy alto pero no hay ninguna duda que Hutchins cuenta con ello y hace aportaciones 
importantes. Presenta una serie de redes neuronales que tienen como objetivo encontrar una simulación que permita explorar las relaciones entre propiedades de individuos y propiedades de grupos. En este sentido Hutchins señala que, dado que el procesamiento en las redes conexionistas se distribuye a través de las unidades en la red, y que el procesamiento en un sistema de cognición socialmente distribuida se distribuye a través de un determinado número de personas, hay una fuerte tentación de pensar que los nodos de una red corresponden a las personas y que las conexiones entre los nodos corresponden a la comunicación entre dichas personas. Si fuera así, una red se tomaría como un modelo de una comunidad pero surgen muchos problemas en este tipo de correspondencia. Hutchins sugiere que:

...el valor real del conexionismo para entender la distribución social de la cognición vendrá de una analogía más complicada en la que los individuos son modelados por redes completas o por conjuntos de redes, y los sistemas de cognición socialmente distribuida son modelados por comunidades de redes. (Hutchins, 1995:248).

No cabe duda de que el recurrir a la ciencia empírica para dar fundamento a la teoría del conocimiento es una forma de naturalizar la epistemología. Sin embargo, no supone una naturalización en sentido fuerte, sino que basta con la tesis mínima sobre naturalización (Estany, 2001) uno de cuyos puntos dice que la psicología cognitiva nos determina cuáles son las mejores condiciones para ejercitar nuestras capacidades cognitivas a fin de optimizar las funciones epistémicas, dejando espacio para la función normativa de la epistemología.

\section{Una nueva forma de abordar los factores sociales}

La CSD introduce los factores psicosociales en la producción de conocimiento. No es la primera vez que se aborda este temá, tanto en el campo de la filosofía como de la antropología, la psicología y la sociología. Sin embargo, hay que señalar que, en la mayoría de los casos, la introducción de factores psicosociales en la producción de conocimiento ha ido asociada a la introducción de irracionalidad y a posturas relativistas. Más recientemente los enfoques que, en una medida u otra, aceptan las tesis del constructivismo social abordan este tipo de problemática pero las consecuencias que sacan de constatar la influencia de los factores psicosociales son todas negativas para la fundamentación del conocimiento. 
También existe una sociología de la ciencia en sentido empirista que estudia las comunidades científicas como grupos humanos en interacción y no saca consecuencias negativas para la epistemología, simplemente hacen sociología de la ciencia como podrían hacer sociología del trabajo o de la familia. Pero tampoco sacan conclusiones positivas ni se plantean la relación entre la interacción social y la epistemología. Merton es uno de los representantes de este enfoque. Lo mismo podríamos decir de los historiadores que se han dedicado a la historia externa de la ciencia pero como algo ajeno a la fundamentación del conocimiento. Por lo tanto, al igual que Merton, tampoco abordan el problema aquí planteado.

La novedad principal de la CSD es que permite rescatar los aspectos positivos del hecho de que el modelo cultural aprendido socialmente intervenga en los razonamientos, cuyo fruto es el conocimiento que los humanos tenemos del mundo.

Algunas de las ideas de Hutchins, pero sobre todo, las de los autores ligados a la actividad situada, pueden parecer que están en la línea del constructivismo social. Mi opinión es que hay diferencias suficientemente importantes como para considerarlos dos enfoques distintos. Hutchins critica no sólo aquellos que piensan que la cognición es manipulación de símbolos formales dentro del cerebro, sino también aquellos que, como Latour en algunos trabajos, ignoran lo que ocurre en el cerebro y tienen en cuenta sólo los procesos externos, pensando erróneamente que en dichos procesos está el lugar de la cultura.

Es cierto que algunos de los trabajos bajo el enfoque del constructivismo social han abordado temas y han planteado problemas que les acerca a la AS y el CSD, sin embargo, ni el marco teórico, ni las consecuencias que han sacado tienen nada que ver, antes bien en algunos puntos son incompatibles. Trabajos como los de Knorr-Cetina (1981) o de Latour y Woolgar (1979) abordan las relaciones entre los miembros de las comunidades científicas pero las consecuencias que sacan es que la comunicación entre los científicos sólo hace acentuar los sesgos procedentes de sus características idiosincráticas, de su procedencia de clase y de su cultura. El trabajo empírico de estos autores está dirigido, fundamentalmente, a demostrar que la ciencia es una construcción social, subjetiva y que, en ningún caso, nos dice cómo es el mundo, ni siquiera aproximadamente.

El mismo Latour en una crítica al libro de Hutchins («Cogito, ergo sumus!: ou l'anthropologie renouvelle la definition de ce que c'est que penser», La Recherche, \#284, Febrero, 1996, p.49), se da cuenta de la distancia que les separa, aunque lo lamenta porque dice que tienen los 
mismos intereses y muchos de los mismos enemigos. Latour se equivoca ya que ni unos ni otros son los mismos. El interés de Latour es demostrar que no es posible el conocimiento del mundo, parte por tanto de una postura absolutamente escéptica y relativista. Por su parte, el interés de Hutchins es justo lo contrario, lo que le interesa es encontrar las estructuras cognitivas más potentes para obtener un mejor conocimiento. Algunos de los enemigos (no todos) pueden ser los mismos, pero no en la misma medida ni por los mismos motivos. Es cierto que ambos se oponen al enfoque cognitivo del procesamiento simbólico pero para Hutchins es una primera etapa que hay que superar, y sigue pensando que los sistemas cognitivos son importantes, aunque difiera con la IA clásica en cuál es la unidad de cognición, y además confia en la ciencia de la computación y ve el conexionismo como un marco fructífero para su proyecto. En cambio, Latour quiere apartarse de la ciencia cognitiva, al menos en su enfoque computacional. En realidad, su relativismo le impide poder confiar en cualquier conocimiento científico.

La relevancia epistémica de las propiedades cognitivas del grupo

La investigación científica nunca se ha realizado en solitario aunque determinados modelos historiográficos hayan impulsado una historia a partir de figuras con nombre propio. Sin embargo, en el momento actual, dado el desarrollo y especialización en la mayoría de las disciplinas científicas, el trabajo en equipo es un requisito imprescindible. Esto significa que en la investigación científica es de capital importancia poder confiar en la información de los miembros del grupo y en la división del trabajo. La investigación científica es pues una actividad cuya unidad de trabajo es el grupo y su función es producir conocimiento del mundo para lo cual se requiere que se ponga en funcionamiento una serie de procesos cognitivos. Por tanto, todo lo referente a la naturaleza y estructura de la cognición del grupo es relevante para la epistemología. La buena nueva para la fundamentación del conocimiento es que el hecho de que la investigación científica se lleve a cabo a partir del grupo puede revertir (insisto en el «puede» porque no necesariamente siempre tiene que ser así) positivamente en la producción y fundamentación del conocimiento.

En este sentido, Hutchins dice que el concepto de división del trabajo es uno de los conceptos más interesantes en antropología, sobre todo en términos del presupuesto de energía de un grupo humano y de la eficiencia con la que este grupo humano explota su entorno físico. Pues bien, la división del trabajo es lo que encontramos en todos los equipos 
de investigación científica y esto va a suponer racionalizar los recursos humanos y técnicos.

Una forma de neutralizar los sesgos de la confirmación

El problema del que partíamos era que no hay un algoritmo para asignar pesos a los valores epistémicos y así establecer una jerarquía entre ellos. La elección, por tanto, está mediatizada por el modelo cultural que interviene en la interpretación de los fenómenos estudiados. Es lo que Hutchins llama «sesgo de la confirmación». Pero, según Hutchins, los modelos de simulación muestran que, incluso manteniendo las propiedades de los individuos constantes, los grupos exhiben propiedades cognitivas distintas en función de cómo se organiza la comunicacjón entre los miembros del grupo. El grupo puede generar una diversidad de interpretaciones que no puede un individuo por sí sólo.

Lo que se desprende de las propiedades cognitivas del grupo es que permite neutralizar los sesgos, revirtiendo en una menor probabilidad de error $y$, por tanto, en una mayor confianza en el conocimiento surgido de la investigación en grupo.

La contribución de la sociología de la ciencia

Hemos visto que las propiedades cognitivas del grupo difieren de las de los individuos que lo forman y que las primeras dependen del tipo de organización. Desde el punto de vista cognoscitivo lo que interesa es ver qué tipo de organización puede convertir en virtud lo que era un vicio a nivel individual. Y es aquí donde la sociología podría hacer aportaciones interesantes a partir de estudios empíricos sobre cuáles son las formas organizativas que favorecen la variabilidad cognitiva, ayudan a neutralizar los sesgos y mantener el equilibrio entre la diversidad interpretativa y la capacidad de llegar a un consenso.

El papel de la metodología de la investigación científica

Si aceptamos la introducción del bagaje sociocultural, la pregunta es hasta qué punto somos prisioneros de nuestra cultura y de nuestro conocimiento anterior. Si los científicos fueran incapaces de salir de su propio esquema conceptual, la objetividad de la ciencia estaría en serios apuros (Estany, 2001). Aquí hay que recordar los parámetros que intervienen en la interpretación de los fenómenos. Uno de estos parámetros es la estructura del constreñimiento subyacente. La metodología de la 
ciencia tendría la función de actuar como las reglas de juego compartidas por los miembros del grupo y que hace que la cognición sea fructílera y haga posible el consenso. Sin estos constreñimientos no sería posible la valoración de las interpretaciones ni posibilidad de comunicación.

No hay que olvidar tampoco que el científico además de pertenecer a una cultura como todos los demás miembros de su sociedad tiene una subcultura que es la cultura científica. Esto significa que la metodología de la ciencia forma parte del bagaje cultural que interviene en la interpretación de los fenómenos.

\section{Conclusiones}

1) El pulso epistémico se produce por la tensión entre valores epistémicos y la razón de esta tensión es que no hay un algoritmo que nos permita saber en cada momento qué valor primar en la investigación científica.

2) Los científicos actúan en función de caracteres idiosincráticos fruto de su historia psicosocial pero bajo constreñimientos impuestos por la metodología de la ciencia.

3) La cuestión está en que la idiosincrasia de los científicos puede jugar en contra de la objetividad de la ciencia, pero la mejor estrategia es aprovechar la variedad cognitiva de los científicos para neutralizar los sesgos.

4) Para ello es necesaria la colaboración entre los científicos y aquí Hutchins nos da un modelo de colaboración cognitiva, que sitúa al grupo como unidad cognitiva y computacional

5) La conclusión última que podríamos sacar respecto a la práctica científica respecto a su aspecto cognitivo es que a pesar de no disponer de un algoritmo en la decisión de teorías, no tenemos por qué dudar de que la filosofía de la ciencia tiene en su haber modelos metodológicos y epistemológicos que los científicos tienen en cuenta y las ciencias empíricas proporcionan modelos cognitivos que explican cómo hacer de la necesidad virtud y poder, así, mantener la objetividad de la ciencia.

\section{BibLiOgRAFía}

AGREE,P.E. \& J.SHRAGER 1991, «Routine Evolution and the Culture of Speed», en Routine Evolution and Culture of Speed (Systems Sciences Laboratory, Xerox Palo Alto Research Center). 
CICOUREL, A. 1994, «La connaissance distribuée dans le diagnostic medical». Sociologie du Travail, 4: 427-449.

CLANCEY,W.J. 1993, «Situated Action: A Neuropsychological Interpretation Response to Vera and Simon». Cognitive Science, 17:87-116.

D'ANDRADE,R. 1989, "Culturally Based Reasoning», en A. Gellatly, D.Rogers y J.A. Sloboda (ed.) Cognition and Social Worlds . Oxford: Oxford University Press.

ESTANY,A., 1990, Modelos de cambio científico. Barcelona: Crítica.

1999, Vida, muerte y resurrección de la conciencia. Análisis filosófico de las revoluciones científicas en la psicología contemporánea. Barcelona: Paidós.

2001, «The Theory-Laden Thesis of Observation in the Light of Cognitive Psychology». Philosophy of Science,

GOLDMAN,A. 1986, Epistemology and Cognition. Cambridge (MA): Harvard University Press.

HEMPEL, C. 1983 «Valuation and Objectivity in Science». En R.S. Cohen y L.Laudan (eds.) Physics, Philosophy and Psychoanalysis, 73-100. Boston: Reidel Publishing Company.

HUTCHINS,E. 1995a Cognition in the Wild. Cambridge (MA): The MIT Press. 1995b «How a Cockpit Temembers its Speeds». Cognitive Science, 19:265-288.

HUTCHINS,E. \& T.KLAUSEN 1996, «Distributed Cognition in an Airplane cockpit», en I.Engeström y D Middleton (ed) Macmillan Publishing Company.

KITCHER,P. 1993, The Advancement of Science. Oxford: Oxford University Press.

KNORR-CETINA, K.D. 1981, The Manufacture of Knowledge: An Essay on the Constructivist and Contextual Nature of Science. Oxford: Pergamon.KUHN,T., 1977, La estructura de las revoluciones científicas. México: Fondo de Cultura Ecónomica.

KUHN,T. 1977, «Objectivity, Value Judgement and Theory Choice». En T.Kuhn, The Essential Tension. Chicago: University of Chicago Press.

LAKATOS,I. 1983, La metodología de los programas de investigación científica. Madrid: Alianza.

LATOUR,B. \& S. WOOLGAR, 1979, Laboratory Life. London: Sage.

LAUDAN, L. 1984, Science and Values. The Aims of Science and their Role in Scientific Debate. Berkeley: University of California Press.

LAVE,J. 1988, Cognition in Practice: Mind, Mathematics and Culture in Everyday Life. Cambridge: Cambridge University Press.

LOZARES,C. (en prensa) «La actividad situada y/o el conocimiento socialmente distribuido», Papers, Barcelona: Universidad Autónoma Barcelona. 
NEWTON-SMITH, W.H. 1987, La racionalidad de la ciencia. Barcelona: Paidós. POPPER,K.R. 1962, La lógica de la investigación científica. Madrid: Tecnos. RUMELHART,D.E., G.HINTON, \& J.L. McCLELLAND 1986 «A general framework for parallel distributed processing». En D.Ru.nelhart \& J.L. McClelland (eds) Parallel distributed processing. Vol.1. Cambridge (MA): The MIT Press.

SUCHMAN,L. 1987, Plans and Situated Actions, Cambridge University Press 1993, «Response to Vera and Simon's Situated Action. A Symbolic Interpretation». Cognitive Science, 17:71-75.

TOULMIN, S. 1977, La comprensión humana. I: El uso colectivo y la evolución de los conceptos. Madrid: Alianza.

VAN FRAASSEN, B. 1980, The Scientific Image. Oxford: Oxford University Press. 